\title{
A Broader Perspective on Three Lifespan Theories: Comment on Boerner and Jopp
}

\author{
Michaela Riediger Natalie C. Ebner \\ Max Planck Institute for Human Development, Berlin, Germany
}

\begin{abstract}
Key Words
Assimilative and accommodative coping • Lifespan theories of developmental regulation - Metamodel of selection, optimization, and compensation · Model of optimization in primary and secondary control
\end{abstract}

Why do some people develop 'better' or 'more successfully' than others? This question has intrigued psychologists and philosophers for a long time. The advancement of modern lifespan psychology in the 1980s has brought momentum to theoretical elaborations of two aspects: what is successful development?, and which regulatory processes underlie successful development? To date, none of these questions has been answered in a way that is generally accepted. Several theoretical accounts have evolved around each [for an overview, see Freund \& Riediger, 2003], often, however, without explicitly relating to each other. Focusing on the second of the aspects - regulatory processes contributing to adaptive development - Boerner and Jopp (this issue) provide one of the rare attempts to systematically analyze and compare contemporary theoretical positions and a selection of relevant empirical findings.

Rather than trying to be extensive - and we agree that less is more in this respect - Boerner and Jopp focus on three of the currently most prominent theoretical approaches: the model of assimilative and accommodative coping (proposed by Brandtstädter and colleagues), the model of optimization in primary and secondary control (OPS; proposed by Heckhausen and Schulz), and the metamodel of selection, optimization, and compensation (SOC; proposed by Paul and Margret Baltes and collaborators). Further in line with a 'less is more' perspective, Boerner and Jopp restrict their comparative efforts to a specific context: how do people deal with major life change and loss? As a result of their analysis, they propose an overarching twoprocess framework that subsumes the developmental-regulatory mechanisms postulated by the three theories under the categories improvement/maintenance and reorientation.

We agree that a comparison of these to date relatively unrelated theories of developmental regulation requires a certain degree of selectiveness and simplification. We also see, however, that this may come at the cost of cutting back the delineation

$\begin{array}{ll}\text { KARGER } & \text { ( 2007 S. Karger AG, Basel } \\ \text { Fax +4161306 12 34 } & \text { 0018-716X/07/0504-0196\$23.50/0 } \\ \begin{array}{l}\text { E-Mail karger@karger.ch } \\ \text { www.karger.com }\end{array} & \begin{array}{l}\text { Accessible online at: } \\ \text { www.karger.com/hde }\end{array}\end{array}$

Michaela Riediger, Center for Lifespan Psychology Max Planck Institute for Human Development Lentzeallee 94, DE-14195 Berlin (Germany) Tel. +493044574 99, Fax +49308249939 E-Mail riediger@mpib-berlin.mpg.de 
of the theories' original scopes and conceptual subtleties. Our aim in this commentary is to take up this concern. In doing so, we hope to complement Boerner and Jopp's selective approach by highlighting a number of considerations from a broader perspective. We start by emphasizing some conceptual refinements that cannot be easily distinguished within the proposed two-process framework. Following that, we present an alternative heuristic for describing interrelations among the three models. This heuristic is based on the models' intended scopes of application, which are different from each other and partly go beyond the contextual frame of major loss and change as chosen by Boerner and Jopp. Finally, we discuss the possibility and usefulness of an overarching theoretical integration of the three models. In agreement with Boerner and Jopp, we conclude that this is not possible to date. It would require a rich and systematic empirical basis of studies testing differential predictions derived from the various theories, which is not yet available.

\section{From General to Specific: Some Details Not to Be Overlooked}

Boerner and Jopp organize their conceptual comparison along a two-process dichotomy of improvement/maintenance versus reorientation. This dichotomy is based on the idea (illustrated by Boerner and Jopp's citation of the serenity prayer) that the three theories overlap insofar as they all propose both engagement and disengagement processes as central developmental-regulatory mechanisms. In our view, this two-process distinction serves the purpose of organizing the theory comparison well. It is parsimonious and highlights conceptual overlap between the models. We argue, however, that it bears the risk of obscuring some important conceptual details as elaborated in the two following examples.

For instance, improvement versus maintenance, subsumed here in one category, refer to motivationally and functionally distinct processes that show differential developmental trajectories across adulthood. Boerner and Jopp imply this by discussing several processes of improvement and maintenance as proposed by two of the models, OPS and SOC (see table 1 in Boerner and Jopp). We argue, however, that this point needs to be made more explicit. To simplify matters and to illustrate our thought, we allude more generally to the widely acknowledged distinction between approach and avoidance motivation and behavioral tendencies. Improvement (e.g., optimization, selective primary control) in this sense corresponds to the approach concept, whereas maintenance (e.g., compensation, compensatory primary control) in the sense of these models broadly translates into the concept of (loss) avoidance. There is ample evidence that approach and avoidance motivation are differentially related to such diverse outcomes as task performance, persistence, and psychological well-being. Furthermore, proponents of both OPS and SOC theory have referred to the approach-avoidance distinction to describe lifespan changes in developmentalregulatory processes. Based on the assumption that younger adults typically have access to resources necessary to acquire skills and improve functioning, but that people encounter fewer opportunities to realize improvement as they get older, these proponents have theorized that improvement orientations should be most salient and adaptive in younger adults, whereas orientations towards maintenance and loss prevention should become more salient and beneficial for high levels of functioning in older individuals [Freund \& Baltes, 2000; Heckhausen, 1997]. Empirical evidence

A Broader Perspective on Three Lifespan Theories
Human Development 2007;50:196-200 
supports these assumptions [Ebner, Freund, \& Baltes, 2006; Freund, 2006; Heckhausen, 1997].

Another example for a conceptual detail that is not obvious in the two-process framework of improvement/maintenance versus reorientation is the notion of selectivity, which is particularly central to the OPS and SOC models. These theories claim that the multitude of developmental pathways that are potentially available for a given individual poses a challenge to developmental regulation. On the one hand, it allows flexible adjustments to different environmental conditions and demands. On the other hand, it bears the risk of diffusing limited resources by investing in too many different developmental options. The challenge, therefore, is to find the right balance between limiting one's potential and overextending oneself. Regulatory processes that address this challenge have been subsumed under the notion of selectivity. As mentioned by Boerner and Jopp, OPS and SOC (in line with other recent theories, such as socioemotional selectivity theory [Carstensen, 1993]) emphasize the fundamental role of selectivity for human development. They posit that focused investment of limited resources into a subset of developmental options provides directionality to development and is necessary for the achievement of higher levels of functioning [Freund \& Baltes, 2000; Heckhausen \& Schulz, 1999]. Proponents of both theories have furthermore argued that the age-associated increase in resource limitations, coupled with the decrease in social-structural scaffolding, render selectivity increasingly necessary and beneficial in older adulthood. Empirical research in various life domains has confirmed the proposed adaptiveness of selectivity, particularly when resources are scarce (e.g., in the case of poor health [Staudinger, Freund, Linden, \& Maas, 1999]), and the proposed age-associated increases in selectivity [Riediger \& Freund, 2006]. Improvement, maintenance, and reorientation endeavors thus need to be selective in order to be adaptive, particularly in older adulthood and in situations where resources are scarce.

In short, the two-process framework suggested by Boerner and Jopp has the advantages of being parsimonious and of emphasizing conceptual overlap among the three models. It masks, however, some conceptual refinements, two of which we have exemplarily illustrated. Next, we will discuss an organizing frame that is independent of specific theoretical propositions and that emphasizes differences between the models - specifically, their intended scopes of application.

\section{A Broader Perspective on the Three Theories: Differences in Scope of Application}

Boerner and Jopp revolve their analysis around a specific context of application, namely major life change and loss. This context is suited to emphasize commonalities among the theories because it falls within the scope of all three models. Boerner and Jopp illustrate this by broadly demonstrating how one and the same behavioral expression (e.g., a particular strategy of coping with vision impairment) is categorized according to each theory. As they point out, this can give rise to confusion: so many different labels for one and the same behavior? In our view, this confusion can be reconciled by bearing in mind that the theories do not just provide different labels for the same phenomena, but that they also differ in their intended scope of application. Boerner and Jopp briefly mention these differences. As we believe that insight 
into the conceptual commonalities of the three models requires an understanding of their respective distinctiveness, we want to further elaborate on this.

The SOC model, developed as a metatheory of developmental regulation, proposes universal mechanisms that are assumed to underlie adaptive development in different periods of the lifespan, across different domains of functioning, and on different levels of analyses (e.g., cell structure, individual, society). The model does not designate any specific content to the SOC processes, which are assumed to have a multitude of different phenotypic manifestations. Proponents of the model have argued that understanding specific implementations of SOC requires embedding the metaprocesses in more specific theories pertaining to the phenomenon of interest. Boerner and Jopp primarily allude to one such contextualization of SOC theory in the domain of setting and pursuing goals [Freund \& Baltes, 2000].

Compared to the general version of the SOC metamodel, the intended scopes of OPS and the model of assimilative and accommodative coping are more specific. The OPS model addresses those processes that make up the individual's ability to control his or her environment and life course. The model of assimilative and accommodative coping focuses, as the name implies, on coping processes, that is, on ways people deal with stressful discrepancies between what is and what should be.

We find it helpful to conceive the application scopes (not the theoretical propositions!) of the three models as nested within each other. This, of course, is a simplifying metaphor. We use it here to facilitate relating the three models to each other. Some phenomena, such as coping with vision loss, fall within the scope of all three theories. Other phenomena, such as planning a career, fall within the scopes of OPS and SOC, but not of assimilative and accommodative coping (at least as long as the individual does not need to cope with considerable failure in goal progress). Still other phenomena, such as neuronal plasticity (e.g., after cortical lesions), only fall within the scope of the SOC metatheory. In the first case, behavioral expressions can be assigned to processes proposed by each of the three models. The contextual frame chosen by Boerner and Jopp is an instance of this first case. Developmental phenomena in the other two cases, in contrast, can be allocated to processes of only one or two of the theories.

Let us emphasize here that we do not mean to imply that the models' level of conceptual specifications are nested within each other. For example, models with more circumscribed scopes are not necessarily more specific in their conceptualizations than models with broader scopes. Even more importantly, the fact that the models' scopes of application may be conceived of as nested within each other does not imply that the models themselves are conceptually nested. There are (partly subtle) conceptual discrepancies, thoroughly described by Boerner and Jopp, which prevent an all-embracing, perfect translatability of one model into the others.

\section{Conclusion: What about Model Integration?}

Boerner and Jopp provide, with much attention to detail, one of the rare systematic comparisons of three current lifespan theories of developmental regulation. Their approach is characterized by an emphasis on overlap among the theories. The first part of our commentary centered on the overarching two-process framework of improvement/maintenance versus reorientation that Boerner and Jopp propose. This

A Broader Perspective on Three Lifespan Theories
Human Development 2007;50:196-200 
framework serves the purpose of organizing the comparison of the models well. Some of the models' conceptual refinements, however, are not obvious in this framework, such as the functional and developmental distinctiveness of improvement versus maintenance processes, or the developmental-regulatory role of selectivity. In the second part of this commentary, we took a broader perspective to complement Boerner and Jopp's approach of confining their analysis to the context of major life change and loss, which constitutes the common denominator in the three theories' scopes. Here we discussed an alternative organizing frame that is independent of specific theoretical propositions and that emphasizes differences in the theories' intended scopes of application.

All these considerations lead to an important question that Boerner and Jopp pose: is an overarching integration of the three models, beyond descriptive comparisons, possible and useful? Boerner and Jopp's conclusion to this question has our agreement: to date, an integration of the three models in the genuine sense is not possible. In our view, this would require a rich base of systematic empirical investigation of differential predictions derived from these theories, which is not yet available. We also agree with Boerner and Jopp that, for now, researchers who want to use these theories as theoretical background in their studies have to decide on one or the other model. We believe that an appropriate fit between the assumed breadth of the phenomenon under investigation and the model's intended scope of application can be an informative criterion for this decision.

\section{References}

Carstensen, L.L. (1993). Motivation for social contact across the life span: A theory of socioemotional selectivity. In J.E. Jacobs (Ed.), Nebraska Symposium on Motivation, 1992: Developmental perspectives on motivation. Current theory and research in motivation. Vol. 40 (pp. 209-254). Lincoln: University of Nebraska Press.

Ebner, N.C., Freund, A.M., \& Baltes, P.B. (2006). Developmental changes in personal goal orientation from young to late adulthood: From striving for gains to maintenance and prevention of losses. Psychology and Aging, 21, 664-678.

Freund, A.M. (2006). Age-differential motivational consequences of optimization versus compensation focus in younger and older adults. Psychology and Aging, 21, 240-252.

Freund, A.M., \& Baltes, P.B. (2000). The orchestration of selection, optimization, and compensation: An action-theoretical conceptualization of a theory of developmental regulation. In W.J. Perrig, \& A. Grob (Eds.), Control of human behavior, mental processes, and consciousness: Essays in honor of the 60th birthday of August Flammer (pp. 35-58). Mahwah: Erlbaum.

Freund, A.M., \& Riediger, M. (2003). Successful aging. In R.M. Lerner, M.A. Easterbrooks, \& J. Mistry (Eds.), Handbook of psychology. Vol. 6: Developmental psychology (pp. 601-628). New York: Wiley.

Heckhausen, J. (1997). Developmental regulation across adulthood: Primary and secondary control of age-related changes. Developmental Psychology, 33, 176-187.

Heckhausen, J., \& Schulz, R. (1999). Selectivity in lifespan development: Biological and societal canalizations and individuals' developmental goals. In J. Brandtstädter, \& R.M. Lerner (Eds.), Action and self-development: Theory and research through the life span (pp. 67-103). Thousand Oaks: Sage.

Riediger, M., \& Freund, A.M. (2006). Focusing and restricting: Two aspects of motivational selectivity in adulthood. Psychology and Aging, 21, 173-185.

Staudinger, U.M., Freund, A.M., Linden, M., \& Maas, I. (1999). Self, personality, and life regulation: Facets of psychological resilience in old age. In P.B. Baltes, \& K.U. Mayer (Eds.), The Berlin Aging Study: Aging from 70 to 100 (pp. 302-328). New York: Cambridge University Press. 